\title{
Novice Rationales for Sketching and Tracing, and How They Try to Avoid It
}

\author{
Kathryn Cunningham \\ University of Michigan \\ Ann Arbor, Michigan, USA \\ kicunn@umich.edu
}

\author{
Shannon Ke \\ Georgia Institute of Technology \\ Atlanta, Georgia, USA \\ shannonke@gatech.edu
}

\author{
Mark Guzdial, Barbara Ericson \\ University of Michigan \\ Ann Arbor, Michigan, USA \\ \{mjguz,barbarer\}@umich.edu
}

\begin{abstract}
Prior research has shown that sketching out a code trace on paper is correlated with higher scores on code reading problems. Why do students sometimes choose not to draw out a code trace, or if they do, choose a different sketching technique than their instructor has demonstrated? In this study, we interviewed 13 CS1 students retrospectively about their decisions to sketch and draw on a recent programming exam. When students do sketch, we find that their sketching choices do not always align with a strict execution of the notional machine. Sketching choices are driven by a search for a program's patterns, an attempt to create organizational structure among intermediate values, and the tracking of prior steps and results. When novices don't sketch, they often report that they've identified the goal that the code achieves. In either case, novices are searching for the functionality of code, rather than merely tracing its behavior.
\end{abstract}

\section{CCS CONCEPTS}

-Social and professional topics $\rightarrow$ Computing education; CS1.

\section{KEYWORDS}

CS1, novice programmers, tracing, sketching, notional machine, external cognition, program visualization

\section{ACM Reference Format:}

Kathryn Cunningham, Shannon Ke, and Mark Guzdial, Barbara Ericson. 2019. Novice Rationales for Sketching and Tracing, and How They Try to Avoid It. In Innovation and Technology in Computer Science Education (ITiCSE '19), fuly 15-17, 2019, Aberdeen, Scotland UK. ACM, New York, NY, USA, 7 pages. https://doi.org/10.1145/3304221.3319788

\section{INTRODUCTION}

When novice programmers solve programming problems, they sometimes use pen and paper. They might draw out variable states, re-write code snippets, or work through calculations. Called sketching [2], annotations [12], or doodles [10], these drawings are an external representation that many students find useful. Research supports the utility of sketching. Sketching is correlated with greater

Permission to make digital or hard copies of all or part of this work for personal or classroom use is granted without fee provided that copies are not made or distributed for profit or commercial advantage and that copies bear this notice and the full citation on the first page. Copyrights for components of this work owned by others than the author(s) must be honored. Abstracting with credit is permitted. To copy otherwise, or republish, to post on servers or to redistribute to lists, requires prior specific permission and/or a fee. Request permissions from permissions@acm.org.

ITiCSE '19, fuly 15-17, 2019, Aberdeen, Scotland UK

(c) 2019 Copyright held by the owner/author(s). Publication rights licensed to ACM ACM ISBN 978-1-4503-6301-3/19/07 . .\$15.00

https://doi.org/10.1145/3304221.3319788 success on code reading problems, with sketched traces being the most successful $[2,10]$.

Prior research on student sketching has involved qualitative and quantitative analysis of scratch sheets. However, the motivations of the sketchers remained a mystery. If sketching is associated with success, why do students only occasionally use it? Why do students sometimes stop part-way through a code trace, behavior that has been associated with particularly low success [2]? Student sketches differ in several ways from the more formal memory diagrams proposed by instructors [7], or the graphics in program visualization tools [19]. We have shown that students' sketches often have a different style than the ones used by their teachers [2]. Students' motivation for why and how they sketch is unclear.

If we knew more about the reasons students sketch and the approaches they favor, perhaps we could design sketching or other visualization techniques that students are more likely to understand, use, interact with, and learn from. In this study, we perform interviews with CS1 students to explore the following:

- Why do novice programmers choose to sketch or not sketch on different problems?

- What rationales do novice programmers provide for their sketching styles?

- Why do novice programmers choose a different sketching technique than their instructor?

\section{BACKGROUND}

\subsection{Tracing and other ways novices read code}

Tracing a piece of code involves tracking memory states over time as the execution of code is simulated. Tracers are running a mental model of the notional machine [17], acting as "human compilers".

We want students to trace code because it can lead to students' greater accuracy during problem-solving [24], and it may play a role in improving learning about the notional machine, a concept where students have many misconceptions [18]. But students do not necessarily trace because it is good for them, and they may take other approaches to solve problems. In this paper, we ask students why they trace code and why they trace the way that they do.

We do know that students voluntarily trace when faced with complex problems. The Leeds Working Group [10] asked students to read and predict the execution of programs that used loops and arrays. Students' scratch paper was collected and analyzed, finding that many students had sketched a code trace.

Fitzgerald et al. [4] performed cognitive walkthroughs with introductory CS students as they solved problems with the same problems used in the Leeds Working Group exam. Using grounded theory, the researchers identified 19 strategies students used while 
solving code reading problems, including close tracing (the most frequent) as well as various types of pattern recognition (e.g., recognizing that this program was like one seen before). However, the use of a certain strategy did not predict success.

Fitzgerald et al.'s interviews have also been analyzed with the SOLO taxonomy as a theoretical lens. Lister et al. found that students were more likely to describe using a multistructural approach, where they discussed the function of multiple pieces of code, rather than a relational approach, where they discussed the code as a whole [11]. Other literature has reported that novices focus on the pieces rather than the program purpose. For example, expert programmers are more likely to use abstract mental representations that focus on the goal the code achieves, while novices are more likely to focus on details of how the code executes [1].

Vainio and Sajaniemi identified factors that they felt inhibited novice code tracing [22]. Tracing requires attention to detail, which can lead to cognitive overload and mistakes (e.g., tracking and using variables) and poor use of external representations. Like Fitzgerald et al.'s pattern recognition, Vainio and Sajaniemi found that students assume functionality from a syntactic structure that matches a previously seen pattern.

\subsection{When and why do students sketch?}

We know that while solving code reading problems, sketching a code trace correlates with success $[2,10]$. However, students are more likely to create these types of sketches on some types of code reading problems than others [12]. For questions that are not about code reading, like Parsons problems [14], students rarely sketch anything [2]. Also, students' sketches often does not use the types of diagrams that instructors teach $[2,23]$.

Analysis of the Leeds Working Group data showed that sketching varied widely from institution to institution, from as as low as $28 \%$ of problems sketched to as high as $92 \%$ [12]. Students consistently sketched more often on code prediction problems than on code completion problems.

Sketches have the potential to not only offload cognition, but also to better coordinate thought [9]. Certainly, sketching out variable states provides a persistent way to track information too complex to remember accurately. But also, in the unconstrained space of a sheet of paper a sketcher can re-arrange information to bring key referents closer together, or make certain information more explicit than it was in a prior form [9].

\subsection{What external representations are common in novice programming?}

The process of code execution, known as the action of the notional machine [3], is not self-evident from code syntax or program output. Systems that teach code tracing attempt to make this challenging, hidden process visible[6, 13, 24].

Software that displays program visualizations is a common approach in the effort to help students form accurate mental models of program execution. Systems like the Online Python Tutor [5] and UUhistle [20] demonstrate the action of the notional machine that a student can step through line by line (see Figure 1a,b). The

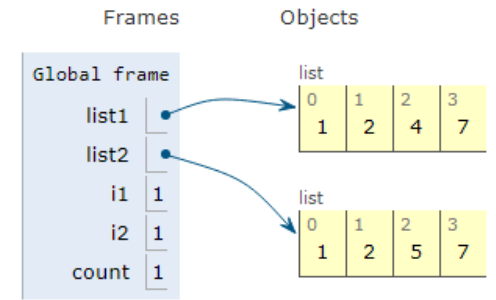

(a) Variables and lists illustrated in Python Tutor [5]

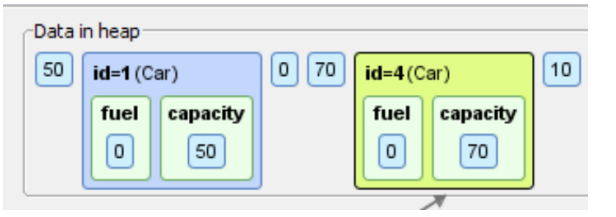

(b) Variables and instances illustrated in UUhistle [20]

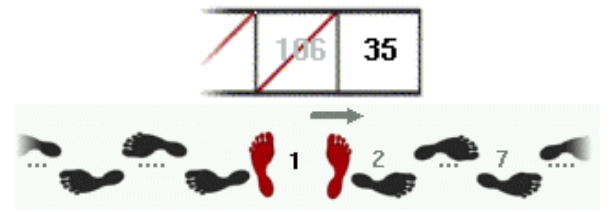

(c) A "most-recent-holder" and a "stepper" variable illustrated in PlanAni [15].

Figure 1: Variable visualizations in program animation tools

visualization style of the great majority of these systems is remarkably consistent in nearly all such systems [19]: variable values are encased in boxes, and prior values are erased and overwritten.

A notable exception is PlanAni [15], a visualization tool that illustrates variables based on their "roles" [16], or functionality. The "most-recent-holder" role and the "stepper" roles are both illustrated with previous values (see Figure 1c).

Empirical studies of visualization systems for use in introductory programming and in algorithms have had inconsistent results $[8,19]$. Visualizations do not always help students, possibly because watching a visualization is a passive learning experience. An interactive process for visual program simulation is more likely to be effective [18]. This may involve an interactive GUI interface, like that of UUhistle [20], or potentially a sketching interface. To that end, viewing visualizations that students create for themselves may suggest new directions for the design of these tools.

\section{METHOD}

CS1 students from a large research university in North America participated in a computer-mediated experiment, using their own laptops in a supervised setting. The experiment took place during the 10 th week of a 16 week semester.

The analysis presented here is from five multiple-choice code reading questions in the pre-test portion of this experiment. These questions were written in Python, and tested CS1 knowledge on lists, loops, and conditionals. In these questions, participants were asked to determine the result after the code had executed. Some questions were examples of common programming plans (see Table 
Table 1: Code plans appearing in code reading questions.

\begin{tabular}{lll}
\hline$\#$ & Apparent code goal & Achieves goal? \\
\hline 1 & Find minimum in a list subset & Yes \\
2 & Find number of common list elements & No \\
3 & Count appearances of target in a list subset & No \\
4 & None (arbitrary arithmetic) & N/A \\
5 & Find average of list subset & Yes \\
\hline
\end{tabular}

1), although the goal of the code was never specified. Students had 15 minutes to answer the five questions.

During the test, participants were instructed to use provided pens and blank scratch paper (labeled with their unique identification number) if they wished to draw. Participants were instructed to return their scratch paper to the experiment administrators after completion of the test.

\subsection{Class observations}

All participants had the same CS1 course instructor. The first author attended two class sessions during weeks 5 and 6, when lists were introduced. Field notes about instructional strategy were taken, and the sketches created by the instructor during these sessions were collected.

\subsection{Interviews}

Twenty-six of 167 test-takers consented to be contacted about a follow-up interview. A subset of those who consented were contacted, in order to maximize the variability the interviewees scores on the pre-test. Twenty-one students were contacted, and 13 agreed to participate in an interview. Of those 13, three got four questions right, six got three questions right, two got two questions right, one got one question right, and one got no questions right.

Interviews were semi-structured, lasted 30 minutes, and occurred in weeks 14,15 , and 16 of the semester.

During the interviews, interviewees' scratch paper from their pre-test was presented, along with the problems from the original exam. Interviewees were given time to re-familiarize themselves with their work, and then asked to describe their choices to sketch or not sketch on each problem. Then, the instructor's sketches were presented, and the students were asked to describe why they chose to sketch similarly or differently than their instructor.

Interviews were recorded and transcribed. They were coded thematically by two coders (the first and third authors), working individually.

\section{WHY SKETCH (OR NOT)?}

\subsection{Goal and pattern recognition}

4.1.1 Identifying a goal. Most questions matched or appeared to match familiar coding plans (see Table 1). For those problems, several students claimed to have identified the goal of the code, and didn't feel a need to sketch anything at all.

Said one interviewee about problem five: "Once you look at the code and figure out what it's doing, then it's like, okay, I can compute an average without writing it down, especially if it's only two values."
Another non-sketcher agreed, with the following thoughts on problem one: "To me, it's very clear that you're looking for a minimum value within a certain range, and so for that, I didn't feel a need to necessarily write down anything."

The opposite was also true. Describing their process for solving problem two, an interviewee recalled how not identifying a goal for the code led to a tracing sketch: "When I saw it I wasn't like 'Oh, I think I have a hunch that this code does this.' I'm going to need to work through it."

4.1.2 Recognizing a pattern. In problems where an overall goal wasn't initially identified, sometimes tracing through a portion of the code execution was enough to identify a pattern and confidently predict the final result. This approach could lead to halting sketching halfway though, as this interviewee described on problem two: "After I sort of got the hang of it, I just started to skip through writing it." An interviewee who abandoned their trace on problem one said: 'You can also see the point where I realized like... and it clicked."

4.1.3 Goal and pattern recognition was not reliable. Despite the confidence expressed by many interviewees in their ability to recognize goals and patterns, they were often wrong. Consistent with the conclusions of Fitzgerald et al. [4], there was significant variety in how well students used the pattern-matching strategy.

\subsection{Anticipated cognitive load}

The complexity of the problem corresponded with the amount of effort put into sketching a trace, with more complex problems sparking more organized tracing approaches. An interviewee recounted their choice to only sketch a few values in an unorganized way as they were solving problem one: "I know it's a bit simpler, I immediately made like an assumption that it was easier, so I didn't put as much work into making a table or a chart."

4.2.1 Complex variable interactions. How is complexity interpreted? Reflecting on their sketching choices for problem four, one interviewee felt that the amount of variable dependencies required a sketched trace: "There were three [variables], I was like, "That's too many," and they're all related. I was like, 'I can't remember that,' and so I drew it out."

4.2.2 Arithmetic or other math-like cues. Math-like cues within code syntax also prompted a move to close tracing: "This [code in problem 4] looks like an equation. All the other ones don't look like equations. I guess when you don't have really long variable names also that might help." Arithmetic was another factor that led to tracing: "Instead of just reassigning values, I'm having to do some basic arithmetic, so keeping track of that through this method [a table] for me was the way to go about it."

\subsection{Problem-solving progression: From goal search to tracing (and re-tracing)}

Rather than immediate use of a tracing technique, interviewees typically described an initial search for code's meaning, then a fall-back to tracing when no discernible goal or pattern was found.

Tracing was repeated two or more times, if an acceptable result was not found. Later tracing attempts were more organized, and more likely to take up a table-like format. In the words of one 


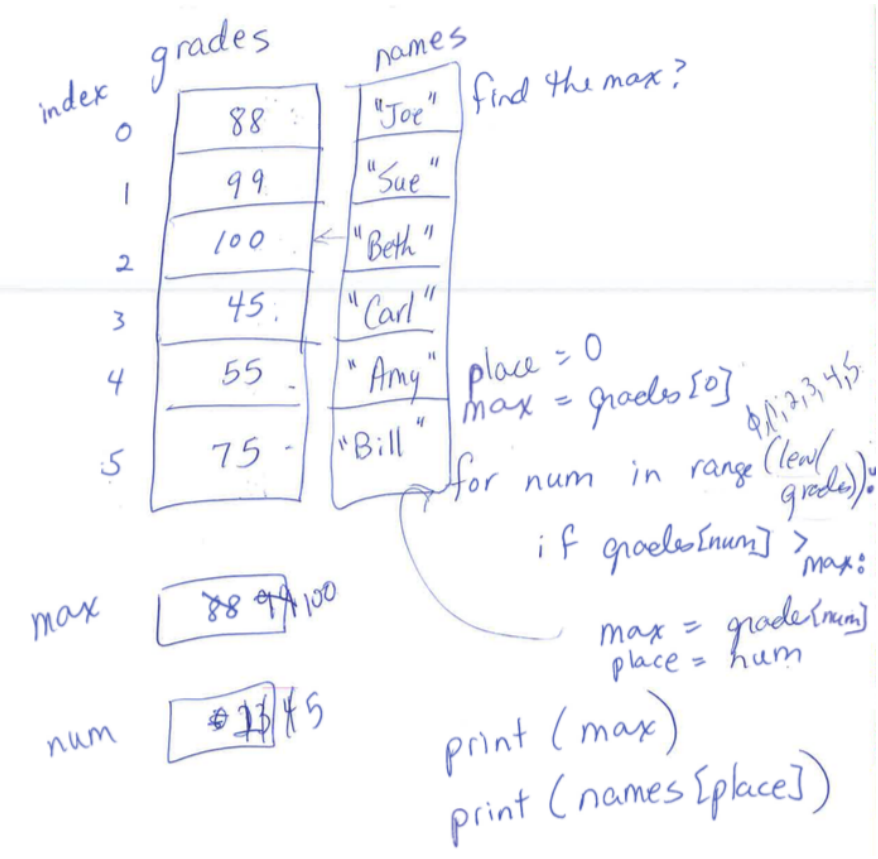

Figure 2: An in-class sketch by the instructor.

interviewee: "When I do a problem, I usually just write stuff fast and try to get through to it. And then if I feel like that's becoming difficult to keep track of everything, I'll slow down and do something like this [a table], to make it easier to see." More organization was often assumed to bring a greater chance of success: "I was kind of annoyed that I didn't get the answer the first time so I was just like okay, putting down lines [creating a table to fill in]. I'm not going to get it wrong this time."

\section{WHY NOT SKETCH LIKE THE INSTRUCTOR?}

The interviewees' instructor used sketched traces in class while teaching about lists and common list procedures, like finding a list's minimum value. One of her illustrations is shown in Figure 2. The instructor's choice to sketch boxes as representations of memory locations aligns with the design of the great majority of program animation software [19] (see Figure 1) as well as proposed designs for sketched memory diagrams (e.g. [6, 21]).

However, no participants re-created this sketching style while problem-solving. Instead, they opted for sketching techniques described in [10] and [2], where memory values are not bounded by boxes, but may be in rows, chunks, or tables. Why didn't students replicate the style repeatedly demonstrated by their instructor?

\subsection{Seen as unnecessary and time-consuming}

Interviewees described this sketching style as not worth the time and effort during exam conditions. "Boxes take too long to draw, basically", said one participant. "I'm always kind of hesitant to diagram things out to the max if I can save time by doing that." "It would take me longer to (draw) the boxes than to write the number, and probably, it will be a mess," said another interviewee.

An interviewee described the instructor's technique as overly detailed, and not prioritizing important values over unimportant ones: "She's trying to illustrate everything as opposed to me just trying to illustrate specifically what I need to get through a problem....she literally writes out every single thing. Even really simple things like if it's a value and it's fixed, she'll write it. Like, it won't change."

Others noted that they didn't see a functional difference in the tracing sketch they performed and what the instructor sketched: "I just didn't circle the numbers, I guess."

\subsection{Visual details seem distant from code}

Some students didn't feel that a box was the most appropriate representation for a variable. Said one interviewee who sketched traces using variable names and equals signs (e.g. $t=1)$ : "I think I just see it a little more clearly as saying that it's a variable that's equivalent to something, as opposed to maybe being a placeholder. I prefer to just write out what it actually is, in terms of a visualization." This student appears hesitant to move away from representations that aren't similar to code syntax.

This prioritization of code was echoed by another participant: "Because the high demand to put code in programming, I feel like, you don't have time to sit there and draw a picture most of the time for a simple list."

\subsection{Boxes are reserved for another purpose}

For two interviewees, boxes had a clear meaning from their use in other subjects: identifying final results. Said one interviewee, "If I cross them out, or box them then I end up getting very confused, because I use boxes and circles for other kinds of notation. I usually box my answers." Pointing at variables in Figure 2, another interviewee said: "I'm guessing those are the initial values but it kind of makes it look like they're the finals. Because from physics, like for homework, I always circle my finals.... saw the box first here and I'm like oh I don't like that."

Boxing a key result, but not intermediate steps, is a technique students likely practiced across years of mathematics and physics courses. These participants didn't want to to change the meaning of this representation.

\section{WHAT DO NOVICE SKETCHES INCLUDE?}

While participants' sketches didn't closely match their instructor's sketches, or the style used in program animation tools, they did show a variety of consistent trends.

\subsection{Organizing and structuring of traces}

6.1.1 Organizing around loop iterations. Sketching out a trace was described as difficult without some sort of organizing structure. The index of the looping variable provided guidance during this cognitively challenging process. As one participant recalled: "I think this one I had to actually do it a couple of times. The first time I went through I actually didn't put the zero, one, two, and three, because that really helped me count the range. Before when I didn't do that I really lost track of what my i value was so it got confusing, so I 


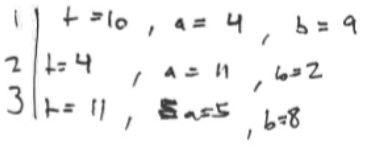

(a) Each row of variable values begins with a loop index

Figure 3: Tracing organized by loop indices

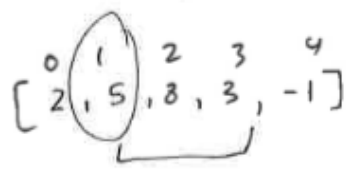

(a) Indice annotations, circling a key value, and demarcating a sub-list

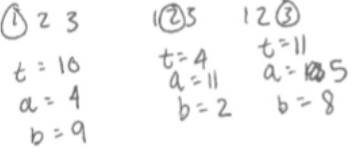

(b) Columns of variable values headed by loop indices

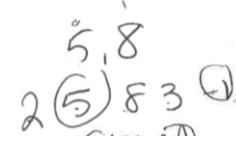

(b) Circling a key value and rewriting sub-list
Figure 4: Tracing organized around a list

erased it a couple of times. Then I put the i values next to it, and it became much more clear."

Describing their sketch (pictured in Figure 3a), another interviewee said: 'It's just for me to establish here's an iteration, here's an iteration, here's an iteration, just so I know what I'm dealing with and then not getting confused between iterations and numbers." This participant's sketch indicates that loop indices are considered a distinct variable type. The loop variables aren't labeled by their variable name, and instead serve to separate parts of the sketched trace.

6.1.2 Organizing around annotated lists. Rather than jumping to a full sketched trace on the questions involving lists, participants used the list itself to organize an abbreviated trace. An interviewee described the creation of such as sketch (pictured in Figure 4): "And then I guess I circled five to start with that. And then I also labeled each value that I knew was probably going to change." Recognizing that the list is key to the question, the interviewee centered their problem-solving there, using the list to get an overview of what occured in the code.

\subsection{Persistence of past values and calculations}

Interviewees were cognizant of the possibility that their sketched trace may need to be re-used or modified. Keeping previous values of variables around for easy reference was a priority, as this interviewee described: "if I mess up, I can go back and know that I either wrote a variable wrong or something like that."

For one participant, this need was enough to dictate their writing utensil: "I like to do coding in pen because if I make a mistake I can clearly see it because I have to cross it. I can't erase it, I have to cross it out." For another, this goal led to careful tallying of the evaluation of conditionals as well as variable values, "so if I ever had to go back through a problem and check my work, I'd be like that's why I did this." Figure $5 \mathrm{~b}$ shows this sketch.

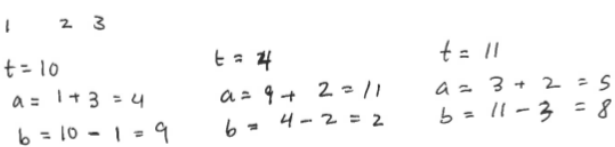

(a) Intermediate values in arithmetic expressions are preserved

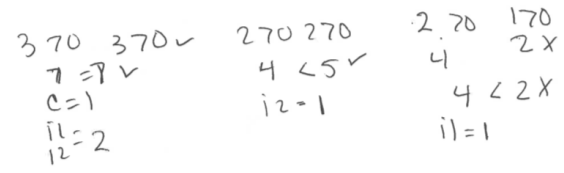

(b) Boolean expression values and variable values are preserved

Figure 5: Persistence of past values

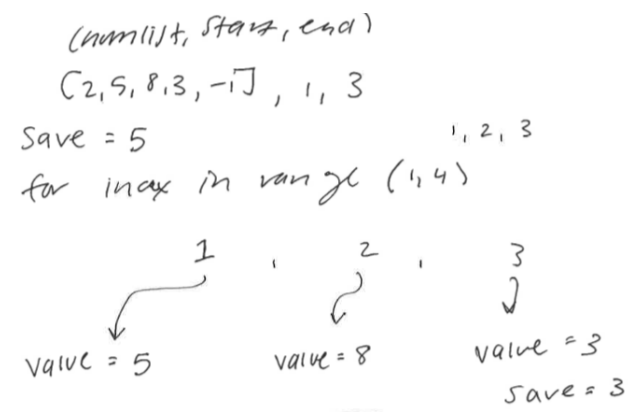

Figure 6: Organizing tracing around code structure

Keeping past values also allowed interviewees to better observe patterns over time. "For me, I think it was just visual and then being able to see it laid out helped me also keep track of it. Because this is going back and forth and iterating it a bunch of times, so seeing the physical changes of the new variables was really helpful."

\subsection{Anchoring with visible values and structures}

6.3.1 Re-writing initial values. Where should students start when faced with a complex code tracing problem? Students often started with initial values. As one interviewee explained: "I always rewrite the first list. It's like for math. I always rewrite the original problem. I know some people don't do that. It takes up a lot of time."

Despite the time commitment, several participants re-wrote starting values, like the arguments passed into the function, or initial variable values.

6.3.2 Using code structure to anchor tracing. Some students rewrote code in a way that oriented their trace, most often for looping structures. In Figure 6, this participant combined the for loop syntax with a trace across three iterations.

\section{DISCUSSION}

Our results identify several problem-solving approaches of CS1 students that differ from the work of a strict "human compiler". 


\subsection{Search for goals and patterns is primary}

These findings suggest that the first task of novice code readers is an attempt to find patterns and goals within code. Novices scan code in an effort to recognize its functionality, or perform an incomplete trace in the hopes of seeing a trend. In both cases, the cognitively demanding work of full, close tracing is avoided.

Such a strategy is understandable, considering the tedium of close tracing. If a student doesn't have a strong understanding of the notional machine, close tracing may be just as error-prone as looking for familiar code structures and divining the functionality from there.

Current visual program simulation approaches connect code structure (the syntax) to code behavior (the action of the notional machine). However, these approaches do not shed light on the function the code is trying to achieve. Can new approaches help learners not only better understand the notional machine, but also more accurately recognize patterns in code?

\subsection{Variables are treated differently based on context}

While a compiler doesn't treat loop variables differently than other variables, novice sketchers do. Loop variables are often used by novices to organize the tracing of other variables. Novices appear to have different classes of variables, based on semantic meaning.

Can visual program simulations treat loop variables differently than other variables? Or, can they use variable names to infer meaning, and emphasize or de-emphasize variable changes accordingly?

\subsection{Past values are retained}

Novice tracers show a preference for retaining previous values as they trace, in order to identify patterns or be able to re-trace their steps.

In program visualizations, the previous value is typically replaced as soon as a new value is assigned to the variable. However, maintaining past values may allow novices to better detect patterns. What effects might retaining some past values in program visualization have on learners?

\subsection{Variables are un-boxed}

Novices are unlikely to maintain a box representation in their own sketched visualizations. The use of boxing may interfere with sketching strategies learned in other courses (like boxing the final value in physics). Or, it may simply be irrelevant when creating a sketch, but still useful when teaching. Visualizing variables in a table-like form creates a more direct opportunity for transfer to a sketching environment.

\section{LIMITATIONS AND THREATS TO VALIDITY}

Our small number of participants allowed for a detailed, qualitative investigation of novice programmer rationales for sketching. However, due to the small sample, we cannot claim any generalizability of our findings. Qualitative research explores experiences or artifacts in rich detail, establishing the existence of phenomena. In order to determine whether these behaviors are replicated by many students, we would need to increase our sample size.
While our findings suggest new possibilities for sketching and visualization techniques, they do not translate into recommendations for classroom practice. We cannot claim that if instructors used the sketching approaches favored by students, better learning will result. Further research with more participants is needed to determine whether certain visualization techniques are typically associated with successful outcomes.

While our participants' sketches served to ground our interviews in past experience, our data ultimately depends on recall based on self-report. Additionally, in the weeks since the exam, participants likely improved their understanding of programming, creating additional challenges to accurate recall.

\section{CONCLUSION}

Sketching is a tangible trail of students' problem-solving process. However, pieces of paper alone can only tell us so much. By talking to novice programmers about their sketches, we identified their motivations for sketching differently than their instructor, for avoiding sketching and tracing, and for organizing their sketching in a variety of styles.

Visualization techniques in the computing education literature are designed by computing experts. Whether in program animation software or sketched by hand, these visualizations emphasize a high fidelity with the way the computer executes code "under the hood". The separation of memory values from code reflects the separation of instruction bytes from data bytes. The box analogy is consistent with parceling of only a certain number of bits per variable. The computer doesn't understand the semantic difference between a loop variable and a counter variable, so they are often illustrated identically.

Novice programmers aren't aware of the "reality" of the machines they are working with, and so while sketching and tracing they mix code and memory values, de-prioritize certain variables, and keep prior values around for reference. Each of these actions supports their ultimate end: the search for goals and patterns in the code they are trying to understand at a human level.

Humans are different than compilers. While a computer must parse and execute code token by token, human learners can infer patterns and identify goals.

Our current visualization techniques don't offer affordances for this type of action. With inspiration from the visualizations novices choose to create for themselves, we may be able to create visualization techniques that students are not only more likely to use, but that could also support their ability to generate meaning from the code they are reading and tracing.

\section{ACKNOWLEDGMENTS}

This material is based upon work supported by the National Science Foundation Graduate Research Fellowship under Grant No. DGC1148903. We also thank the University of Michigan for financial support.

\section{REFERENCES}

[1] Beth Adelson. 1984. When novices surpass experts: The difficulty of a task may increase with expertise. Journal of Experimental Psychology: Learning, Memory, and Cognition 10, 3 (1984), 483. 
[2] Kathryn Cunningham, Sarah Blanchard, Barbara Ericson, and Mark Guzdial. 2017 Using Tracing and Sketching to Solve Programming Problems: Replicating and Extending an Analysis of What Students Draw. In Proceedings of the 2017 ACM Conference on International Computing Education Research (ICER '17). ACM, New York, NY, USA, 164-172. https://doi.org/10.1145/3105726.3106190

[3] Benedict Du Boulay. 1986. Some Difficulties of Learning to Program. Fournal of Educational Computing Research 2, 1 (1986), 57-73. https://doi.org/10.2190/ 3LFX-9RRF-67T8-UVK9

[4] Sue Fitzgerald, Beth Simon, and Lynda Thomas. 2005. Strategies That Students Use to Trace Code: An Analysis Based in Grounded Theory. In Proceedings of the First International Workshop on Computing Education Research (ICER '05). ACM, New York, NY, USA, 69-80. https://doi.org/10.1145/1089786.1089793

[5] Philip J. Guo. 2013. Online Python Tutor: Embeddable Web-based Program Visualization for Cs Education. In Proceeding of the 44th ACM Technical Symposium on Computer Science Education (SIGCSE '13). ACM, New York, NY, USA, 579-584. https://doi.org/10.1145/2445196.2445368

[6] Matthew Hertz and Maria Jump. 2013. Trace-Based Teaching in Early Programming Courses. Proceedings of the 44th ACM Technical Symposium on Computer Science Education (2013), 561-566. https://doi.org/10.1145/2445196.2445364

[7] Mark A. Holliday and David Luginbuhl. 2004. CS1 assessment using memory diagrams. ACM SIGCSE Bulletin 36, 1 (2004), 200. https://doi.org/10.1145/1028174. 971373

[8] Christopher D. Hundhausen, Sarah A. Douglas, and John T. Stasko. 2002. A MetaStudy of Algorithm Visualization Effectiveness. Fournal of Visual Languages and Computing 13, 3 (2002), 259-290. https://doi.org/10.1006/S1045-926X(02)00028-9

[9] David Kirsh. 2010. Thinking with external representations. Ai \& Society 25, 4 (2010), 441-454

[10] Raymond Lister, Otto Seppälä, Beth Simon, Lynda Thomas, Elizabeth S. Adams, Sue Fitzgerald, William Fone, John Hamer, Morten Lindholm, Robert McCartney, Jan Erik Moström, and Kate Sanders. 2004. A multi-national study of reading and tracing skills in novice programmers. In ACM SIGCSE Bulletin, Vol. 36. 119-150. https://doi.org/10.1145/1041624.1041673

[11] Raymond Lister, Beth Simon, Errol Thompson, Jacqueline L Whalley, and Christine Prasad. 2006. Not seeing the forest for the trees: novice programmers and the SOLO taxonomy. ACM SIGCSE Bulletin 38, 3 (2006), 118-122.

[12] Robert McCartney, Jan Erik Moström, Kate Sanders, and Otto Seppälä. 2004 Questions, Annotations, and Institutions: observations from a study of novice programmers. In the Fourth Finnish/Baltic Sea Conference on Computer Science Education, October 1-3, 2004 in Koli, Finland. Helsinki University of Technology, Department of Computer Science and Engineering, Laboratory of Information Processing Science, FINLAND, 11-19.

[13] Greg L Nelson, Benjamin Xie, and Andrew J Ko. 2017. Comprehension first: evaluating a novel pedagogy and tutoring system for program tracing in CS1. In
Proceedings of the 2017 ACM Conference on International Computing Education Research. ACM, 2-11.

[14] Dale Parsons and Patricia Haden. 2006. Parson's Programming Puzzles: A Fun and Effective Learning Tool for First Programming Courses. In Proceedings of the 8th Australasian Conference on Computing Education - Volume 52 (ACE '06). Australian Computer Society, Inc., Darlinghurst, Australia, Australia, 157-163. http://dl.acm.org/citation.cfm?id=1151869.1151890

[15] Jorma Sajaniemi. 2002. PlanAni-a System for Visualizing Roles of Variables to Novice Programmes. University of Joensuu, Department of Computer Science.

[16] Jorma Sajaniemi and Marja Kuittinen. 2005. An experiment on using roles of variables in teaching introductory programming. Computer Science Education 15, 1 (2005), 59-82.

[17] Juha Sorva. 2013. Notional Machines and Introductory Programming Education. Trans. Comput. Educ. 13, 2, Article 8 (July 2013), 31 pages. https://doi.org/10. $1145 / 2483710.2483713$

[18] Juha Sorva et al. 2012. Visual program simulation in introductory programming education. Aalto University.

[19] Juha Sorva, Ville Karavirta, and Lauri Malmi. 2013. A Review of Generic Program Visualization Systems for Introductory Programming Education. ACM Transactions on Computing Education 13, 4 (2013), 15.1 - 15.64. https://doi.org/10.1145/ 2490822

[20] Juha Sorva and Teemu Sirkiä. 2010. UUhistle: A Software Tool for Visual Program Simulation. In Proceedings of the 10th Koli Calling International Conference on Computing Education Research (Koli Calling '10). ACM, New York, NY, USA, 49-54. https://doi.org/10.1145/1930464.1930471

[21] Lynda Thomas, Mark Ratcliffe, and Benjy Thomasson. 2004. Scaffolding with Object Diagrams in First Year Programming Classes: Some Unexpected Results. In Proceedings of the 35th SIGCSE Technical Symposium on Computer Science Education (SIGCSE '04). ACM, New York, NY, USA, 250-254. https://doi.org/10. $1145 / 971300.971390$

[22] Vesa Vainio and Jorma Sajaniemi. 2007. Factors in Novice Programmers' Poor Tracing Skills. SIGCSE Bull. 39, 3 (June 2007), 236-240. https://doi.org/10.1145/ 1269900.1268853

[23] Jacqueline Whalley, Christine Prasad, and P. K. Ajith Kumar. 2007. Decoding Doodles: Novice Programmers and Their Annotations. In Proceedings of the Ninth Australasian Conference on Computing Education - Volume 66 (ACE '07). Australian Computer Society, Inc., Darlinghurst, Australia, Australia, 171-178. http://dl.acm.org/citation.cfm?id=1273672.1273693

[24] Benjamin Xie, Greg L Nelson, and Andrew J Ko. 2018. An explicit strategy to scaffold novice program tracing. In Proceedings of the 49th ACM Technical Symposium on Computer Science Education. ACM, 344-349. 